\title{
Online Healthcare Consultation System for Deaf \& Dumb During Pandemic Situation
}

\author{
Sampada Wazalwar ${ }^{{ }^{*}}$ and Urmila Shrawankar ${ }^{2}$ \\ ${ }^{1}$ Research Scholar, ${ }^{1,2}$ Computer Science \&t Engineering \\ ${ }^{1,2} \mathrm{G}$ H Raisoni College of Engineering, Nagpur, India
}

\section{ABSTRACT}

Pandemic situations knocks at the door without information. In such situation healthcare is a major concern. Increased count of patients in any adverse condition leads to scarcity of healthcare services. In such situation telemedicine has played a very important role to overcome health problems of patient \& to promote the remote healthcare monitoring of patients by physician in online mode. This proved to be very helpful for normal people but physically challenged could not take its advantage because of disabilities. We have implemented a system to reduce the communication barrier. System include many modules most importantly sign recognition \& sign interpretation module in combination with ISL database of medical terms. For sign recognition we are using HMM model with Haar cascade classifier. Interpretation system fully uses the language technology including POS tagging, parsing etc. The overall system proved to improve the accuracy \&t efficiency of online consultation system for Deaf \& Dumb people.

KEY WORDS: TELEMEDICINE, HEALTHCARE, CONSULTATION, SIGN RECOGNITION, SIGN INTERPRETATION, SIGN LANGUAGE, SPOKEN LANGUAGE, COVID19

\section{INTRODUCTION}

Telemedicine is now becoming an important part of everyone's life. It is gaining more and more importance due to its advantages, time scarcity with people. Remote health monitoring is also a part of it. We have experienced its benefits during this today's world pandemic as well. As it has helped to monitor the patient's condition remotely during isolation period. Otherwise also when people are scared of going in hospitals because of pandemic condition, this technology helps a lot to the patients as well as physician for continuous health monitoring. Thanks to the technology today which is growing rapidly

\section{ARTICLE INFORMATION}

*Corresponding Author: sampadawazalwar@gmail.com Received 18th Oct 2020 Accepted after revision 29th Dec 2020 Print ISSN: 0974-6455 Online ISSN: 2321-4007 CODEN: BBRCBA

Thomson Reuters ISI Web of Science Clarivate Analytics USA and Crossref Indexed Journal

\section{Clarivate
Analytics}

NAAS Journal Score 2020 (4.31)

A Society of Science and Nature Publication,

Bhopal India 2020. All rights reserved.

Online Contents Available at: http//www.bbrc.in/

Doi: http://dx.doi.org/10.21786/bbrc/13.14/50 to increase comfort of human life. But, there are still some community in society which are still not using or unable to use such technology for health monitoring. Here we are specially talking about the physically challenged people like Deaf \& Dumb. Deaf \& dumb cannot take the advantage of remote healthcare monitoring services because of communication problem. Telemedicine also involves direct online appointment booking \&t video communication with physicians where a patient can show \&t explain his problem to physician directly. Deaf \& dumb cannot do this because of communication language as sign language is not known to all $\mathrm{Ct}$ even there is also restrictions for medical terms in sign language dictionary.

Use of assistive technology is increasing day by day. There are many assistive technologies in terms of hearing aid, speech to text, text to speech, image to text, smart blind sticks, smart goggles for blind \& many other android applications [Emad, 2016] etc for helping disabled in day to day life. Our system includes sign language interpretation in meaningful phrase. It also makes the 
things understandable to the physicians as well. There is a possibility that people can communicate the things to physicians by writing but, explaining so many things in writing also becomes difficult after a period of time. Verbal communication is always easy in comparison with written communication. But, in case of Deaf \& Dumb, other person should also have a knowledge of Sign language which is basically a challenge. Implemented health monitoring or consultation system for Deaf \& Dumb includes a sign interpretation module as well. This proves very much helpful in pandemic situations like COVID19, we all eye witnessed.

Current Scenario: Telemedicine for healthcare is gaining more and more importance because of technological advances \&t its benefits for the patients. Benefits of telemedicine includes that patient need not to visit hospital repeatedly which reduces travelling time \& cost. Patient need not to remain bedside for small problems. 24*7 physician's consultation is available. There are many current applications which are helpful for remote monitoring. Some applications are for medicine supplies as well. COVID19 pandemic has increased the use of such applications amongst people at great level. Many applications are used for different purposes like appointment booking, online consultation, home delivery of medicine service \&t pathological services etc. Various applications includes Physicianlink , Netmeds, Mfine, Practo, Medlife \&t Ayushman Bharat.

These systems proved to be very useful during COVID 19 pandemic situation where people preferred to be at their home. Many hospitals are also having their own online consultation services. But, here we are specially talking about the facilities available for Deaf \& Dumb for assistance in healthcare for remote monitoring, there are no such applications available. Sign language is a visual language, which is difficult to understand without any knowledge. Thus, there is always a need of sign recognition \&t interpretation system which will translate sign language to spoken language in understandable phrases. So, during pandemic situation though the world is taking help of telemedicine applications, Deaf $\mathrm{t} t$ Dumb community could not found solution. Physician's consultation can also be taken telephonically for normal people. But, for deaf $\mathrm{Ct}$ dumb it becomes necessary to have a video communication.

\section{Remote Health Monitoring system for Deaf \& Dumb METHODOLOGY}

Deaf \& Dumb community uses sign language for communication. It is difficult for any normal to directly communicate to Deaf \& Dumb and vice versa because of language barrier $\&$ the thoughts remains unshared. Healthcare is a sector where it is the right of every person to take advantage of all facilities. Using today's technological advances and smart algorithms we made a system for Deaf \& Dumb. We added some modules to existing system which are used for sign interpretation. Implementation of such system has following challenges
- Physicians knowledge about sign language

- Patients (who is Deaf Ct dumb) understanding of spoken language

- Sign language medical term dictionary

- Sign recognition accuracy

- Interpretation system in between

- Continued internet connection with good resolution camera

In this system both ways interpretation is very essential. The prior need is physician should understand the symptoms which are communicated by Deaf \& Dumb patient and in return patient should understand the consultation given by physician. In some cases physician may send the advice by writing if deaf \& dumb is well aware of spoken language, but if it is not the case then reverse translation from spoken to sign also become essential, especially when that Deaf \& Dumb person do not have anyone to assist or he is in isolation condition. Proposed system model is shown in the fig 1 below. We are proposing fully software system which will be user friendly. The system may also be included dictionary separately where a patient can learn the sign for medical term if he is not having knowledge of it. There still many terms in medical which do not have any sign in such cases fingerspelling or character by character representation can be done to spell the word.

Figure 1: System model for remote healthcare monitoring for Deaf \& Dumb

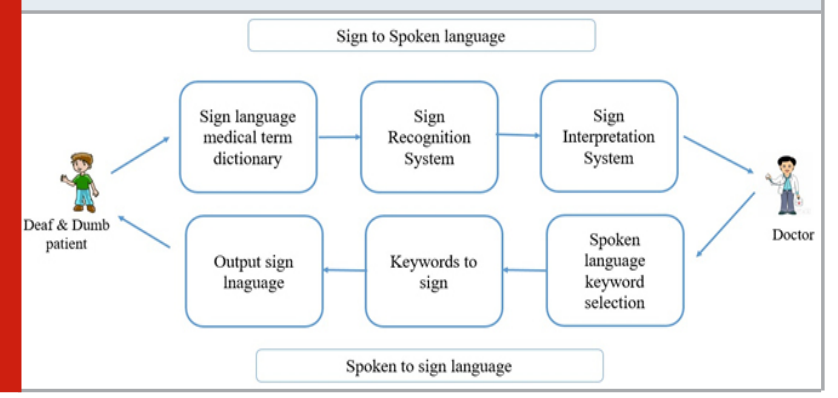

The model for remote healthcare monitoring application for Deaf \& Dumb includes two way conversions. Start from Sign language to spoken language for patient to physician communication \&t other from spoken language to sign language for physician to patient communication. There are many different proposed sign recognition systems. Some are hardware based written here [S. Mitra, 2007] \&t some are software based written here [Masrur Sobhan, 2019]. Of course wearing hardware for this is not comfortable so a software system can only help in this model. The overall performance of model depends on the accuracy of translation system which depends on training dataset. As here we are basically talking about healthcare, system needs to be trained for some standard signs for medical terms.

Now the question comes for the data set. Indian Sign Language Research \&t Training Centre (ISLRTC) has released ISL dictionary of 3000 words in March 2018. The second edition also released in February 2029. It includes 
6000 words under the categories of academic, legal, medical, technical and everyday terms. So the overall dictionary includes approximately 500 medical terms which includes standard signs for basic medical terms which are needed to communicate medical problems. The examples of medical terms included in dictionary are listed below.

Table 1. Sample keywords in medical dictionary of ISL

\begin{tabular}{|l|l|l|l|}
\hline \multicolumn{5}{|c|}{ Example of Medical terms in ISL } \\
\hline headache & fever & cough & Cold \\
\hline swallow & stomach & pain & itching \\
\hline Crack & bones & vomit & loss \\
\hline Blood & mouth & teeth & Joint \\
\hline Nose & Eyes & Tongue & Hands \\
\hline Blood & vein & Brain & Spinal cord \\
\hline Memory & Skin & faint & Injection \\
\hline Blood Pressure & Pulse & Heart rate & Thermometer \\
\hline Oximeter & ventilator & Heart attack & cancer \\
\hline
\end{tabular}

Figure 2: System Flow Diagram

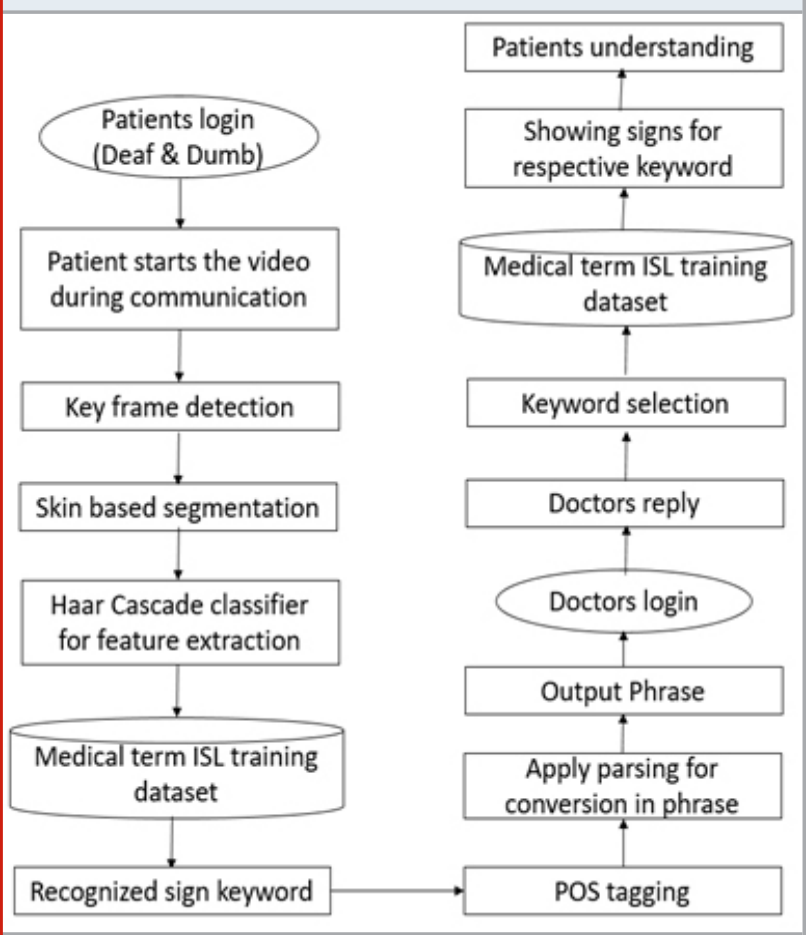

In addition to above many other terms including each body part are also there in ISL dictionary. The application needs the knowledge of both the corpus for translation. Spoken language like English corpus is easily available At the ISL corpus which is quite difficult. Medical term database is available online in terms of videos. So the overall system includes following steps to complete the conversation.

\section{Implementation Details}

Step 1: Patient \& physician login to system

Step 2: Patient \&t physician needs to start video communication
Step 3: Application will capture the gesture of patient \&t apply sign recognition using feature extraction, key frame identification, gesture identification and assigning the sign keyword for that gesture by system training.

Step 4: Recognized sign keywords will be given as input to sign interpretation system which will apply POS tag on it and after applying parsing system will output phrase.

Step 5: Output will be understandable phrase for physician

The detail implementation of system is given in flowchart below. Flowchart shown in figure 2 represents the detail steps in system. The system mainly concentrates on understanding the problem of Deaf \& Dumb. This will make consultation easy for physicians.

Table 2. Input keywords for recognized patient gesture and output phrase

\begin{tabular}{l|l}
\multicolumn{1}{c|}{ Input identified keywords } & \multicolumn{1}{c}{ Output phrase } \\
\hline Me fever & I am having fever / I have fever \\
\hline Experience headache & I am experiencing headache \\
\hline Pain toes & I have pain in toes \\
\hline Me recover & I am recovered \\
\hline Need rest & You need rest \\
\hline Tell symptoms & Tell me the symptoms \\
\hline How you now & How are you now? \\
\hline Tell history & Tell me your history \\
\hline What name & What is your name? \\
\hline Where pain & Where do you experience pain \\
\hline
\end{tabular}

\section{RESULTS AND DISCUSSION}

The system helps to translate the sign language into spoken language to ease the communication between physician \& Deaf \& Dumb patient for remote consultation during pandemic situations. Sign language do not have the standard grammar \& it has many limitations as compared to spoken language. Thus, we expect the results in following form considering selected sign keywords will be interpreted in the form of understandable meaningful phrase. Following table shows the input \& system generated output sentences. Table 2 below shows some list of input keywords \& system interpreted output. It helps physician to understand what patient wants to say \& it also avoid the misinterpretation which is very important point of consideration in healthcare consultation.

\section{CONCLUSION}

Implemented system gives 85\% accurate results. Accuracy is calculated by comparing output of system with actual human interpreter. The system proved to be helpful for physician \& patient in emergency situation. Similar system can also be used for many other purpose like online or remote education for Deaf \& Dumb. It will motivate them to share their thoughts \&t knowledge by recording their own video where it will 
be automatically getting interpretation in English for others understanding.

\section{ACKNOWLEDGEMENTS}

We want to acknowledge following people for providing valuable inputs for designing our system as per need today $\&$ according to their experience with Deaf $\&$ Dumb, during communication $\&$ consultation.

1. Dr. Harshal Khobragade, MBBS, MD, Sr. Resident at Lata Mangeshkar Hospital, Nagpur

2. Dr. Kalyan Wazalwar, Consultant Pediatrician \&t Neonatologist at Shri Shankaracharya Institute of Medical Sciences, Bhilai (CG)

3. Principle \& Teachers of Deaf \& Dumb School, Shankar Nagar, Nagpur

\section{REFERENCES}

Emad Abdallah, Ebba Fayyoumi,Assistive Technology for Deaf prople based on android platform, procedia computer science 94, science direct, 2016, 295-301. http://www.islrtc.nic.in/isl-dictionary-launch https://www.physicianlink.com/

Masrur Sobhan, Mehrab Zaman Chowdhury et.al, A Communication Aid System for Deaf and Mute using Vibrotactile and Visual Feedback, 2019 International Seminar on Application for Technology of Information and Communication (iSemantic), DOI: 10.1109/ ISEMANTIC.2019.8884323

S. Mitra and T. Acharya, "Gesture Recognition: A Survey, systems, man \& cybernetics, Part C: Applications \& Reciews”, IEEE Transactions on, 2007. D0I:10.1109/ TSMCC.2007.893280

Sampada S. Wazalwar, Urmila Shrawankar, "Interpretation of sign language into English using NLP techniques", Journal of Information and Optimization Sciences, 38:6, 895-910, DOI: 10.1080/02522667.2017.1372136.

Urmila Shrawankar, Azra Shireen, Suggesting Teaching Methods by Analysing the Behaviour of Children with Special Needs, Bio-Algorithms and Med-Systems, Volume 16, Issue 1, 20190038, ISSN (Online) 1895-9091 | 1896-530X , Pages 1-20, DOI: 10.1515/bams-20190038, Publisher ^ 20207. 